\title{
Consumo de tabaco en estudiantes universitarios: motivación a la cesación y dependencia ${ }^{1}$
}

\author{
Francisco-Javier León ${ }^{2}$, Giampaolo Orlandoni-Merli³, Yenny-Carolina Bernal-Luna ${ }^{4}$, \\ Fanny-Rosmira Gómez-Balaguera ${ }^{5}$, Laura-Patricia Amaya-Díaz ${ }^{6}$
}

\section{Resumen}

Introducción. En 2018, el cáncer de pulmón se ubicó en primer lugar a nivel mundial, tanto en incidencia y mortalidad con 2,0 y 1,8 millones de casos respectivamente. Además, en los últimos cuatro años, ocupó el cuarto lugar en prevalencia, con 2,1 millones de casos anuales. Objetivo. Determinar la dependencia y motivación a la cesación del consumo de tabaco en una muestra de estudiantes universitarios mediante pruebas estandarizadas y entrevista semi-estructurada. Materiales y métodos. Se trata de un estudio mixto, transversal. Para evaluar la dependencia a la nicotina y la cesación del consumo se emplearon las pruebas de Fagerström y Richmond. Resultados. Se observa ausencia de relación entre el grado de motivación para la cesación del consumo de tabaco y el nivel de dependencia. Los sujetos presentan un nivel dudoso de dejación del consumo de tabaco a pesar de encontrarse en una dependencia muy baja. El factor familiar y social se convierte en promotor de la dependencia e iniciación. Conclusión. Se identifica una baja motivación para la dejación del consumo, por lo tanto, es necesario promover intervenciones dirigidas a la atención del tabaquismo.

Palabras clave: adicción, cáncer, cese del tabaquismo, dependencia, motivación

1 Artículo original derivado del proyecto de investigación "Efecto genotóxico causado por la exposición y consumo de tabaco en estudiantes de los programas de salud de las sedes de Bucaramanga y Valledupar de la Universidad de SantanderUDES", ejecutado entre febrero de 2016 al febrero de 2018; Grupo de investigación Ciencias Básicas y Aplicadas para la Sostenibilidad - CIBAS. Financiado por la Vicerrectoría de Investigaciones de la Universidad de Santander - UDES, acta de inicio número 055-15. Agradecimientos a COLCIENCIAS por el apoyo a la Joven Investigadora en el marco de la convocatoria 743 Jóvenes Investigadores e Innovadores 2016, contrato número 410, derivado del trabajo de grado presentado por Fanny-Rosmira Gómez-Balaguera.

2 Maestría en Ciencias Básicas Biomédicas, Bacteriólogo y Laboratorista Clínico, Docente Asociado Universidad de Santander, miembro del Grupo de investigación Ciencias Básicas y Aplicadas para la Sostenibilidad - CIBAS, Correo: fleon@udes.edu.co, ORCID https://orcid.org/0000-0002-8904-5242

3 Doctor en Estadística, Maestría en Economía, Economista. Docente Titular, Universidad de Santander, miembro del Grupo de investigación Ciencias Básicas y Aplicadas para la Sostenibilidad - CIBAS, correo: gorlandoni@udes.edu.co, ORCID https://orcid.org/0000-0002-0031-2659

4 Medica, miembro del Grupo de investigación Ciencias Básicas y Aplicadas para la Sostenibilidad - CIBAS. Universidad de Santander, correo: yenny.bernalluna@gmail.com, ORCID https://orcid.org/0000-0002-8136-6623,

5 Psicóloga, miembro del Grupo de investigación Ciencias Básicas y Aplicadas para la Sostenibilidad - CIBAS. Universidad de Santander, correo: ynnaf1214@hotmail.com, ORCID https://orcid.org/0000-0001-5779-230X,

6 Psicóloga, Especialista en Psicología Clínica. Maestría en Educación. Docente Asistente, Universidad de Santander. Miembro del grupo de investigación estudios socio-humanísticos, correo: lau.amaya@mail.udes.edu.co, ORCID https:// orcid.org/0000-0001-9103-7594

Autor para correspondencia: Francisco Javier León, correo: fleon@udes.edu.co www.udes.edu.co Recibido: 20/12/2018 Aceptado: 21/07/2020 


\section{Tobacco consumption in university students: motivation to the cessation and dependence}

\section{Uso do tabaco em estudantes universitários: motivação para cessação e dependência}

Abstract

Introduction. In 2018, lung cancer ranked first worldwide in both incidence and mortality with 2.0 and 1.8 million cases respectively. In addition, in the last four years, it ranked fourth in prevalence, with 2.1 million cases per year. Objective. To determine tobacco dependence and motivation to quit in a sample of university students through standardized tests and semistructured interviews. Materials and methods. This is a mixed, cross-sectional study. Fagerström and Richmond tests were used to evaluate nicotine dependence and cessation. Results. There is no relationship between the degree of motivation for smoking cessation and the level of dependence. The subjects presented a doubtful level of tobacco cessation despite being in a very low dependency. The family and social factor becomes a promoter of dependence and initiation. Conclusion. A low motivation for quitting consumption is identified, therefore, it is necessary to promote interventions aimed at tobacco care.

Keywords: addiction, cancer, smoking cessation, dependency, motivation

\section{Resumo}

Introdução. Em 2018, o cancro do pulmão ocupava o primeiro lugar mundial em incidência e mortalidade, com 2,0 e 1,8 milhões de casos, respectivamente. Além disso, nos últimos quatro anos, classificou-se em quarto lugar em prevalência, com 2,1 milhões de casos por ano. Alvo. Determinar a dependência do tabaco e a motivação para deixar de fumar numa amostra de estudantes universitários através de testes padronizados e entrevistas semi-estruturadas. Materiais e métodos. Este é um estudo misto, de corte transversal. Os testes Fagerström e Richmond foram utilizados para avaliar a dependência e cessação da nicotina. Resultados. Não existe qualquer relação entre o grau de motivação para deixar de fumar e o nível de dependência. Os sujeitos apresentaram um nível duvidoso de cessação do tabaco apesar de se encontrarem numa dependência muito baixa. $\mathrm{O}$ factor familiar e social torna-se um promotor de dependência e de iniciação. Conclusão. Identifica-se uma baixa motivação para deixar de consumir, pelo que é necessário promover intervenções que visem os cuidados com o tabaco.

Palavras chave: vício, câncer, abandono do uso de tabaco, dependência, motivação,

ocupado el cuarto lugar con 1.026.877 casos (Globocan, 2018; Services et al., 2014). Los datos anteriores reflejan un problema de salud pública de múltiples orígenes siendo uno de ellos el consumo de cigarrillo o tabaco en la adolescencia (Romero et al., 2017) o en entornos de formación universitaria de profesionales de la salud de acuerdo con investigaciones desarrolladas por (Pinilla-Vásquez \& Angarita- 
Fonseca, 2012; Services et al., 2014; Warren et al., 2011; Yang et al., 2015).

El tabaco posee múltiples sustancias cancerígenas especialmente en el humo, ya sea para el consumidor directo o para las personas expuestas (Centers for Disease Control and Prevention (US) \& U.S. Department of Health and Human Services, 2006; Hang et al., 2013). Otras sustancias como la nicotina se asocian con la dependencia dado que tienen efecto en el sistema nervioso central generando cambios en el estado de ánimo, disminución de la ansiedad, cambios de comportamiento, y supresión del apetito; los fumadores expresan que les reduce el estrés, aumenta el rendimiento durante la ejecución de tareas que se asocian con procesos psicológicos básicos (Kendler et al., 1999; World Health Organization, 2004). Otros estudios evidencian que el tener familiares o amigos del entorno cercano consumidores de tabaco, el escaso nivel educativo de los padres y el consumo de alcohol se convierten en factores motivantes para el inicio del consumo de cigarrillo, mientras que los factores predictores se observan a nivel psicológico cuando existe pérdida de autocontrol, en la búsqueda de nuevas sensaciones, al presentar momentos de impulsividad y cuando se tienen dificultades en el procesamiento de emociones (Romero et al., 2017). De igual manera, cambios a nivel contextual como el inadecuado uso del tiempo libre, problemas en la atmósfera familiar, relación con pares que presenten conducta antisocial, fracaso escolar y problemas externalizantes durante la adolescencia han sido considerados predictores de consumo (Romero et al., 2017; Villena Ferrer, 2009).

Considerando los elementos anteriores se hace necesario para el diagnóstico de la dependencia al consumo de tabaco estudiar factores familiares, sociales, genéticos, ambientales, culturales y propios del comportamiento del individuo (Kendler et al., 1999; Organización
Mundial de la Salud, 2020; Villena Ferrer, 2009) en ese sentido la Clasificación Internacional de Enfermedades y Problemas de Salud Conexos (CIE-10) cataloga los efectos del tabaco y la nicotina como un trastorno de la categoría «efecto tóxico de otras sustancias no especificadas» [T 65.2]. Según la CIE-10 por dependencia se entiende un conjunto de manifestaciones fisiológicas, comportamentales y cognoscitivas asociadas al consumo de una sustancia, la cual adquiere la máxima prioridad para el individuo y cuya manifestación es el deseo constante de consumirla. Los criterios para el diagnóstico sugieren la existencia de dependencia física, psicológica y social como elementos complementarios.

En lo que respecta a la intención de fumar, Bermúdez \& Contreras, (2008) exponen la teoría de comportamiento planificado (theory of planned behaviour-TPB) donde se "sostiene que, a mayor actitud, apoyo social y percepción del control de la conducta, mayor será la probabilidad de tomar la decisión y llevarla a cabo"; en este sentido se han estudiado el arrepentimiento anticipado y las normas sociales dentro de la TPB, trabajos enfocados en las intenciones y las medidas de estabilidad de la intención en relación con el inicio del hábito de fumar (Conner et al., 2006; Lazuras et al., 2012a). Por el contrario, el modelo transteórico de cambio supone la existencia de tres componentes: los estadíos, los procesos y los niveles; así por ejemplo Prochaska et al., (1994) han planteado los siguientes estadíos de cambio respecto al abandono del consumo de tabaco: precontemplación (la persona no reconoce la conducta de fumar como un problema), contemplación (el fumador empieza a ser consciente de que existe un problema), preparación (el fumador tiene la intención de empezar el proceso de cambio y acción) y actuación (la persona consigue el cambio en su conducta problema) (Prochaska \& DiClemente, 1983). 
El consumo de tabaco genera dos tipos de dependencia: dependencia física que se relaciona con la nicotina, sustancia responsable del síndrome de abstinencia, y dependencia psicológica, que se observa cuando este hábito ocurre en diversas situaciones de la vida diaria, como son, al despertar, después de las comidas, al tomar bebidas, en reuniones, entre otras; por lo cual, investigadores como Fagerström et al., (1978) desarrollaron una prueba para evaluar la dependencia a la nicotina que años después fue ajustada por Heatherton et al., (1991). Esta prueba ha sido aplicada en diversos trabajos de investigación (de Meneses-Gaya et al., 2009; Fu et al., 2011; Radzius et al., 2003; Ussher et al., 2016). La prueba de Fagerström se ha complementado con la medición de sustancias bioquímicas producto del metabolismo del consumo de tabaco (Etter, 2005; Fidler et al., 2011; Fu et al., 2011; John et al., 2004; Valles Vegas et al., 2011)

Para medir la motivación de la dejación del consumo de tabaco la más empleada es la prueba de Richmond, que evalúa características propias del tratamiento y que predice el resultado para la abstinencia continua a los 3, 6 y 12 meses (Richmond et al., 1993). Esta prueba se emplea de forma individual o en conjunto con la prueba de Fagerström (Kotz et al., 2013; Piñeiro et al., 2016; SepúlvedaSánchez et al., 2018; Valles Vegas et al., 2011). Otros estudios documentan procesos de intervención de la dejación y los resultados son analizados con técnicas estadísticas como la regresión logística multivariable, con el fin de establecer la asociación entre la abstinencia y diferentes factores que motivan el consumo (Coleman et al., 2002; Joly et al., 2017; Raherison et al., 2005).

De acuerdo con lo anterior, se planteó un estudio piloto mediante la aplicación de pruebas estandarizadas a una muestra de estudiantes universitarios para determinar su dependencia y motivación a la cesación del consumo de tabaco, confrontándose luego esas pruebas con entrevistas semiestructuradas hechas a los mismos estudiantes muestreados.

\section{Materiales y métodos}

\section{Diseño}

Se realizó un estudio bajo el enfoque mixto (cuantitativo - cualitativo), y para esto se utilizaron datos de corte transversal.

\section{Participantes}

La población estuvo conformada por 796 estudiantes de un programa medicina de una universidad del oriente colombiano. Se calculó el tamaño de la muestra basándose en un muestreo no probabilístico por conveniencia, y se conformó la muestraúnicamente con estudiantes fumadores de todos los semestres del programa.

\section{Pruebas estandarizadas}

Para evaluar la dependencia a la nicotina se empleó la prueba de Fagerström (Heatherton et al., 1991) la cual está conformada por seis modalidades. Para este trabajo se utilizó la modificación realizada por Valles Vegas et al., (2011) como consecuencia de conteos bajos en algunas modalidades. La puntuación empleada se presenta en la tabla 1.

Tabla 1. Modalidades de la prueba de Fagerström y su puntuación.

\begin{tabular}{lc}
\hline \multicolumn{1}{c}{$\begin{array}{c}\text { Modalidades prueba } \\
\text { Fagerström }\end{array}$} & Puntuación \\
\hline Dependencia muy baja & $0-1$ \\
\hline Dependencia baja & $2-3$ \\
\hline Dependencia moderada & $4-5$ \\
\hline Dependencia alta & $6-7$ \\
\hline
\end{tabular}




\begin{tabular}{cc}
\hline $\begin{array}{c}\text { Modalidades prueba } \\
\text { Fagerström }\end{array}$ & Puntuación \\
\hline Dependencia muy alta & $8-10$ \\
\hline
\end{tabular}

Fuente: Adaptada de Valles Vegas et al., 2011

La prueba de Richmond se utilizó para medir la cesación del consumo de tabaco y está estructurada con cuatro modalidades y se asigna una puntuación entre 0 y 10, tabla 2 (Richmond et al., 1993).

Tabla 2. Modalidades de la prueba de Richmond y su puntuación.

\begin{tabular}{lc}
\hline \multicolumn{1}{c}{$\begin{array}{c}\text { Modalidades prueba } \\
\text { Richmond }\end{array}$} & Puntuación \\
\hline $\begin{array}{l}\text { Motivación nula o baja para dejar } \\
\text { de fumar }\end{array}$ & $<3$ \\
\hline Motivación dudosa & $4-5$ \\
\hline Motivación moderada & $6-7$ \\
\hline Motivación alta & $8-10$ \\
\hline
\end{tabular}

Fuente: Adaptada de Richmond et al., 1993.

En cuanto a la entrevista semiestructurada, ésta se estructuró con ocho preguntas organizadas en tres modalidades: condiciones de inicio de consumo, condiciones de mantenimiento del consumo y motivación para la cesación. Estas modalidades determinan los factores psicosociales que mantienen la dependencia y/o la motivación para la dejación del consumo de tabaco (León et al., 2019).

\section{Procedimiento}

Se cumplió con la normatividad para el desarrollo del estudio en concordancia con lo planteado en la Resolución 8430 de 1993 y la Ley 1090 del 2006, entre las cuales se encuentra la aplicación del consentimiento informado, el mantenimiento de la privacidad y la devolución de resultados. Para la aplicación de las pruebas se contó con el aval del comité de bioética de la institución y la aprobación de los directivos del programa. Para la convocatoria de los estudiantes se seleccionó un curso por cada semestre. El tiempo promedio de aplicación de las pruebas fue de 5 minutos. Como criterio de inclusión se consideró que el estudiante fuera consumidor de tabaco; el criterio de exclusión fue ser estudiante de otros programas y en este punto se resaltó que la participación era voluntaria, anónima y confidencial.

\section{Análisis de datos}

Se hizo un análisis estadístico descriptivo a las bases de datos resultantes de las pruebas estandarizadas, además de pruebas de independencia de las variables, mediante test de chi cuadrado de Pearson; luego se hizo análisis factorial exploratorio con rotación varimax para encontrar los factores subyacentes. En lo que respecta al análisis general de las entrevistas, se transcribieron y codificaron en categorías simples y subcategorías relacionadas con los factores psicosociales. Posteriormente se llevó a cabo el recuento y concurrencia de códigos, comparación, contextualización e interpretación. El análisis de los datos se realizó utilizando el programa Statgraphics Centurion XV.

\section{Resultados}

En el $29^{\circ}$ Simposio Internacional de Estadística (2019) se presentó un adelanto de este trabajo, donde mostraron los resultados obtenidos hasta ese momento, y que se evidencian en las tablas estadísticas 3 y 4 , publicadas en las memorias del congreso (León et al., 2019). Las pruebas estandarizadas se aplicaron al 7,16\% (57) de los estudiantes del programa; posteriormente, al $35 \%$ (20) de los fumadores participantes se les realizó entrevista semiestructurada para conocer qué aspectos psicosociales estaban relacionados con el consumo y la cesación. En cuanto al sexo de los participantes, el 77,2 \% fueron hombres 
con una edad media de 23 años (DE $\pm 4,3)$. Las mujeres conformaron el $22,8 \%$ de la muestra, con una edad media de 21 años (DE $\pm 32,2)$.

\section{Prueba de Fagerström y prueba de Richmond}

El $8,8 \%$ de los estudiantes presentan dependencia moderada, el 28,1\% dependencia baja y el 63,2 \% dependencia muy baja a la nicotina, según la prueba de Fagerström. Mientras que la prueba de Richmond evidencia que $14,1 \%$ de los estudiantes presentan motivación nula, el 40,6 \% motivación dudosa, el 22,8\% motivación moderada con necesidad de ayuda, y el 22,8 \% motivación alta a salir del consumo. Los resultados de la prueba Fagerström y la prueba de Richmond son independientes de las variables género y edad $(p>0,05)$.

\section{Análisis factorial exploratorio aplicado a los resultados de la prueba de Fagerström}

El análisis factorial revela que la varianza total explicada por los tres primeros factores, luego de rotación varimax, es $31,5 \%, 22,8$ $\%$ y $15,1 \%$ respectivamente. Los factores comunes después de realizar rotación varimax se muestran en la tabla 3 y en la figura 1.

Tabla 3. Análisis factorial de la prueba de Fagerström.

\begin{tabular}{lccc}
\hline \multicolumn{1}{c}{ Prueba de Fagerström } & \multicolumn{3}{c}{ Factor } \\
\cline { 2 - 4 } & $\begin{array}{l}\text { Persistencia } \\
\text { F1 (31,5 \%) }\end{array}$ & $\begin{array}{c}\text { Mantenimiento } \\
\text { F2 (2,8 \%) }\end{array}$ & $\begin{array}{c}\text { Frecuencia } \\
\text { F3 (15,1 \%) }\end{array}$ \\
\hline $\begin{array}{l}\text { FP1. ¿Cuánto tiempo pasa entre que se levanta y fuma } \\
\text { su primer cigarrillo? }\end{array}$ & $-0,04$ & $\mathbf{0 , 7 1}$ & $-0,03$ \\
\hline $\begin{array}{l}\text { FP2. ¿Encuentra difícil no fumar en lugares donde está } \\
\text { prohibido, como la biblioteca o el cine? }\end{array}$ & 0,27 & $\mathbf{0 , 6 2}$ & 0,25 \\
\hline FP3. ¿QQué cigarrillo le molesta más dejar de fumar? & $\mathbf{0 , 8 9}$ & $-0,04$ & $-0,03$ \\
\hline FP4. ¿Cuántos cigarrillos fuma cada día? & $-0,04$ & 0,07 & 0,98 \\
\hline $\begin{array}{l}\text { FP5. ¿Fuma con más frecuencia durante las primeras } \\
\text { horas después de levantarse que durante el resto del día? }\end{array}$ & $\mathbf{0 , 8 6}$ & 0,18 & $-0,01$ \\
\hline $\begin{array}{l}\text { FP6. ¿Fuma, aunque esté tan enfermo que tenga que } \\
\text { guardar cama la mayor parte del día? }\end{array}$ & 0,04 & $\mathbf{0 , 8 0}$ & 0,02 \\
\hline
\end{tabular}

Método Extracción: componentes principales. Método Rotación: Varimax con normalización Kaiser. Medida adecuación de muestreo $\mathrm{KMO}=0,56$ (porcentaje de varianza explicada).

Fuente: elaborado por los autores. Memorias $29^{\circ}$ Simposio Internacional de Estadística (2019).

La ecuación del primer factor rotado, que refleja la persistencia en las pruebas de Fagerström, es la siguiente F1 $=-0,04 \mathrm{FP} 1+0,27$ FP2 + 0,89 FP3-0,04FP4+ 0,85 FP5 + 0,04 FP6.
El segundo factor representa el mantenimiento, y el tercer factor es una manifestación de la frecuencia con que se fuma. 
Figura 1. Análisis factorial con rotación Varimax para la prueba de Fagerström.

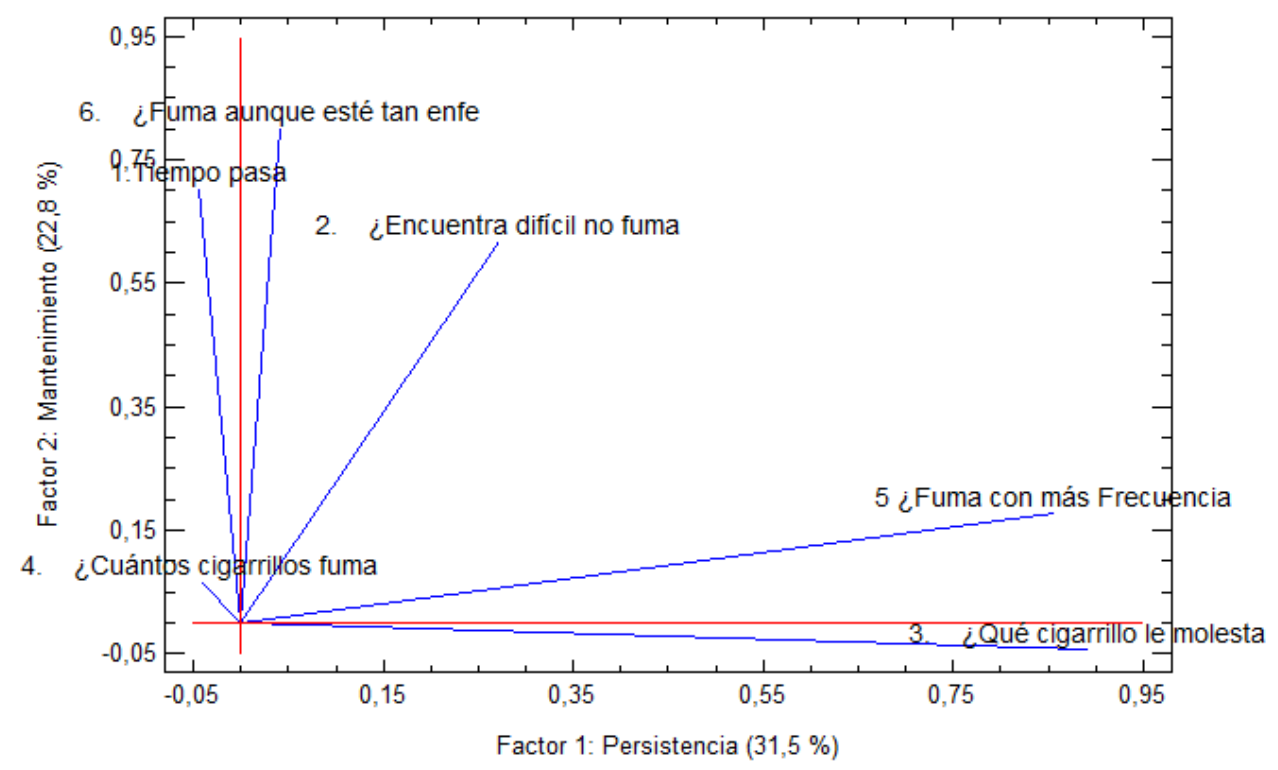

Factores F1: Persistencia y F2: Mantenimiento.

Fuente: elaborado por los autores.

\section{Análisis factorial exploratorio aplicado a los resultados de la prueba de Richmond.}

Luego de rotación varimax, la varianza total explicada por los tres primeros factores obtenidos del análisis de los resultados de la prueba de Richmond, es de 50,0 \%, 27,7 \% y $13,3 \%$ respectivamente, como se muestra en la tabla 4 y la figura 2.

Tabla 4. Análisis factorial prueba de Richmond.

\begin{tabular}{lccc}
\hline \multirow{2}{*}{\multicolumn{1}{c}{ Prueba de Richmond }} & \multicolumn{3}{c}{ Factor } \\
\cline { 2 - 4 } & $\begin{array}{c}\text { M. Dejación } \\
\text { F1 (50,0 \%) }\end{array}$ & $\begin{array}{c}\text { Autoeficacia } \\
\text { F2 (27,7 \%) }\end{array}$ & $\begin{array}{c}\text { Cesación F3 } \\
\mathbf{( 1 3 , 3 \% )}\end{array}$ \\
\hline RP1. ¿Le gustaría dejar de fumar si pudiera hacerlo fácilmente? & 0,15 & $\mathbf{0 , 9 8}$ & $-0,05$ \\
\hline RP2. ¿Con qué ganas quiere dejarlo? & $\mathbf{0 , 8 4}$ & 0,31 & 0,12 \\
\hline RP3. ¿Intentará dejar de fumar en las próximas dos semanas? & $\mathbf{0 , 8 8}$ & $-0,01$ & 0,26 \\
\hline RP4. ¿Cree que dentro de 6 meses no fumará? & 0,24 & $-0,05$ & $\mathbf{0 , 9 7}$ \\
\hline
\end{tabular}

Método Extracción: componentes principales. Método Rotación: Varimax con normalización Kaiser. Medida adecuación de muestreo $\mathrm{KMO}=0,60$ (porcentaje de varianza explicada).

Fuente: elaborado por los autores. Memorias $29^{\circ}$ Simposio Internacional de Estadística (2019). 
La ecuación del primer factor rotado, que representa motivación a la dejación para la prueba de Richmond es F1 = 0,15 RP1 + 0,84 RP2 + 0,88 RP3 + 0,24 RP4. El segundo factor se interpreta como la autoeficacia, mientras que el tercer factor es una manifestación de la cesación a fumar por parte de los estudiantes que conformaron la muestra de estudiantes analizados.

Figura 2. Análisis factorial con rotación Varimax para el prueba de Richmond.

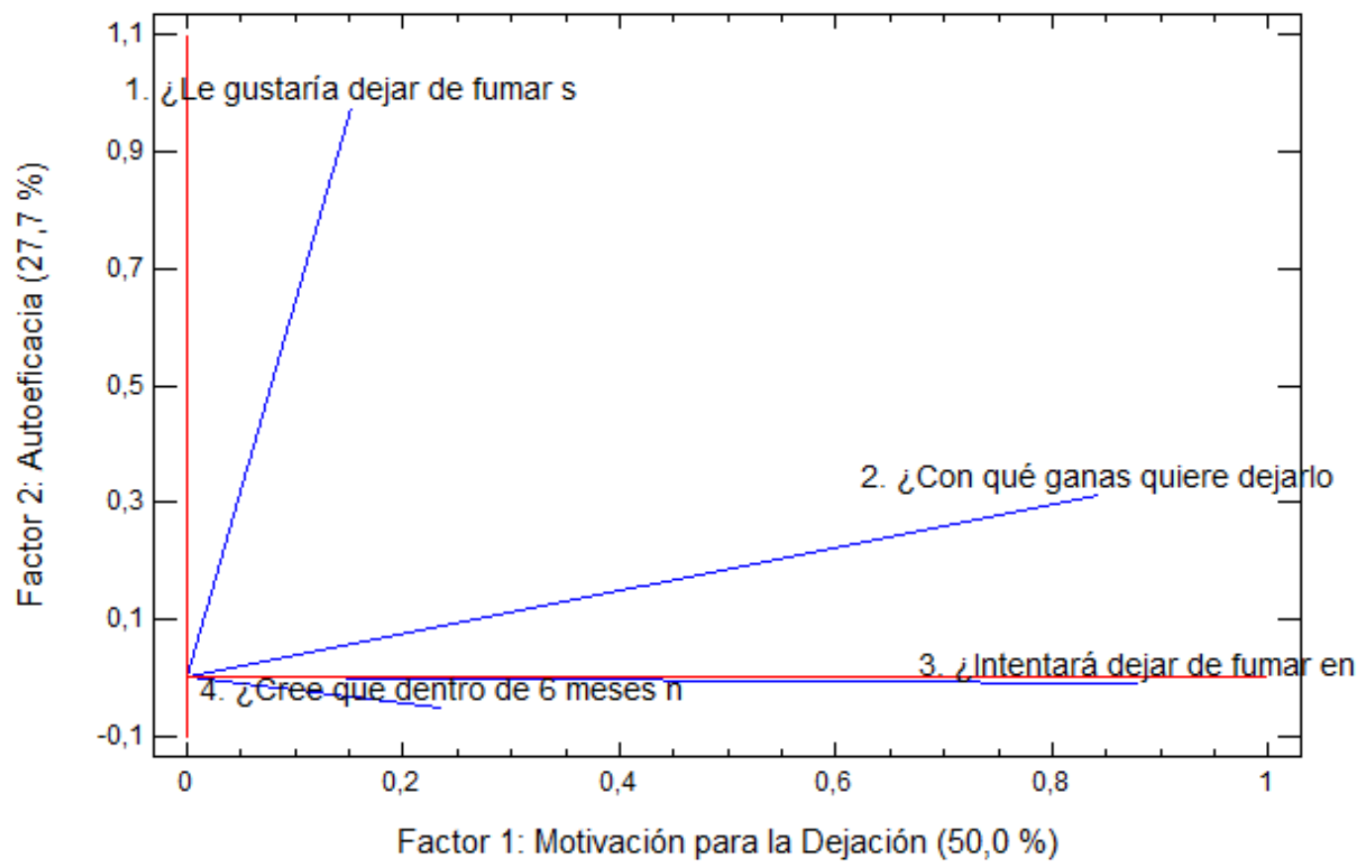

Factores F1: Dejación. F2: Autoeficacia.

Fuente: elaborada por los autores.

\section{Análisis general de las entrevistas}

En la tabla 5 se describen los resultados de las entrevistas semiestructuradas las cuales permitieron identificar los factores asociados al inicio del consumo, los contextos relacionados con mayor frecuencia de consumo y las razones que motivan a los estudiantes a fumar. Las verbalizaciones evidencian que el factor social es el más importante, seguido del familiar y el personal. Respecto a las estrategias para evitar la dependencia, se encuentran tres principalmente: la primera relacionada con sustitución de la nicotina por elementos como el cigarrillo electrónico, los chicles y los alimentos. La segunda es evitar personas y contextos que activan la necesidad de consumir y la tercera consiste en solicitar asistencia profesional. 
Tabla 5. Factores y contextos asociados al consumo.

\section{Factores asociados al inicio del consumo}

\begin{tabular}{|c|c|c|}
\hline Categoría & Subcategoría & Verbalizaciones \\
\hline Social & $\begin{array}{l}\text { Presión social } \\
\text { Imitación } \\
\text { Aprobación social }\end{array}$ & $\begin{array}{l}\text { "Fue porque unos amigos me dijeron y ya después pues sí me } \\
\text { terminó gustando " }\end{array}$ \\
\hline Familiar & $\begin{array}{l}\text { Influencia de modelos } \\
\text { familiares } \\
\text { Imitación de modelos }\end{array}$ & $\begin{array}{l}\text { "Porque tengo un primo que también fuma, pues hay mucha } \\
\text { gente en mi familia que fuma" }\end{array}$ \\
\hline Personal & $\begin{array}{l}\text { Curiosidad de experimentar } \\
\text { sensaciones }\end{array}$ & "Un día salimos y me dio curiosidad de probar de fumar y ya" \\
\hline \multicolumn{3}{|c|}{ Contextos asociados a mayor frecuencia de consumo } \\
\hline Social & Fiestas & "Cuando estoy en fiestas" \\
\hline Familiar & Dificultades en las relaciones & "En referencia a mi novia, peleas con mi novia" \\
\hline Académico & $\begin{array}{l}\text { Parciales } \\
\text { Presión académica }\end{array}$ & "Cuando tengo bastante presión académica" \\
\hline Personal & $\begin{array}{l}\text { Dificultad para dormir } \\
\text { Sentimientos de tristeza y } \\
\text { aburrimiento } \\
\text { Posterior a las comidas } \\
\end{array}$ & $\begin{array}{l}\text { "Sobre todo después de alguna comida, sobre todo después del } \\
\text { almuerzo" } \\
\text { "Cuando tengo tristeza o rabia, cuando estoy en situaciones diría } \\
\text { yo donde se ve involucrado las emociones" }\end{array}$ \\
\hline \multicolumn{3}{|c|}{ Factores que motivan el consumo de tabaco } \\
\hline Social & Fiestas, reuniones sociales & $\begin{array}{l}\text { "La fiesta está aburrida porque es como donde mayormente lo } \\
\text { hago y salgo como para despejar la mente" }\end{array}$ \\
\hline Familiar & Búsqueda de relajación & "Para relajarme, por no sé por distraerme" \\
\hline Personal & $\begin{array}{l}\text { Sentimiento de tristeza, rabia, } \\
\text { euforia y ansiedad }\end{array}$ & $\begin{array}{l}\text { "Pues básicamente el estrés, y la presión que estoy manejado } \\
\text { a mi alrededor, la verdad siento que es mi polo a tierra en esos } \\
\text { momentos que me dice como okey, no va a pasar nada y vamos } \\
\text { a hacer todo racionalmente" }\end{array}$ \\
\hline
\end{tabular}

Fuente: elaborada por los autores.

\section{Discusión}

La mayoría de los estudiantes de la muestra presentaron dependencia muy baja al tabaco similar a otros estudios (Fernández et al., 2015; Lazuras et al., 2012b; Pérez-Milena et al., 2006; Valles Vegas et al., 2011). Una menor proporción mostraron dependencia moderada comparada con el estudio de Rodríguez Gázquez et al., (2010). Como factor de mantenimiento se encontró que los estudiantes usan el consumo de tabaco para sentirse relajados y ser aceptados por sus pares. Según Gonzálvez et al., (2015) un factor predictor de intensidad y frecuencia del consumo de tabaco está dado por el estado de ánimo bajo, lo que ocasiona que los estudiantes de la muestra tiendan a incrementar el consumo de tabaco.

En lo que respecta la prueba de Richmond se evidencia un nivel de motivación dudosa similar a los hallazgos del estudio realizado 
con estudiantes de Medicina en Argentina por Fernández et al., (2015) y contrario al estudio de Castaño-Castrillón et al., (2008) quienes obtienen una baja o moderada motivación para abandonar este hábito por parte de los fumadores.

Con respecto al análisis factorial con rotación varimax para la prueba de Fagerström se identificaron tres factores: el F1 representa la persistencia, el F2 indica el mantenimiento y el F3 revela la frecuencia mientras que en el estudio de Radzius et al., (2003) y Roa-Cubaque et al., (2016) se obtienen solo dos factores. Para la prueba de Richmond, se obtuvieron también tres factores: el F1 muestra la motivación a dejar de fumar y comprende determinados elementos situacionales intrínsecos que mediante la ejecución logran un incentivo. E1 F2 representa la autoeficacia, definida por Bandura (1977) como «la convicción que uno tiene de que puede realizar con éxito la conducta requerida para producir los resultados apetecidos» (Coleman et al., 2002). El F3 muestra la cesación a fumar observada cuando el individuo logra abstenerse de fumar por un tiempo de seis o más meses.

En cuanto a los factores psicosociales, los hallazgos se asocian con la teoría del aprendizaje social de Bandura (1986), la cual sustenta que las personas aprenden mediante observación e imitación del comportamiento de pares o referentes importantes (Feist et al., 2014). Al confrontar los hallazgos de las pruebas y las entrevistas se encontró que el factor social determina el inicio del consumo de tabaco en los estudiantes y ocurre en entornos de confianza (familiares o grupos de amigos), hallazgos similares a las investigaciones realizadas por Pérez-Milena et al., (2006), (2012) quienes concluyen que el inicio y mantenimiento se genera por la identificación en las relaciones con pares y la presencia del hábito en familiares, influencia la conducta de los jóvenes, lo que convierte el factor familiar en un promotor de la dependencia o iniciación del consumo. Además, al asociarse con disminución de la ansiedad es empleado como estrategia de afrontamiento ante situaciones evaluadas como amenazantes (León et al., 2019).

Según Cifuentes et al., (2009) las experiencias de familiares fumadores influyen directamente en la toma de decisiones de los jóvenes, pues genera una actitud positiva o negativa frente al consumo, como lo demostraron Alfonso et al., (2009); el apoyo familiar puede conducir a una disminución en el consumo de tabaco, a diferencia de familias disfuncionales donde la influencia de pares (hermanos, primos y tíos de la misma edad del consumidor de tabaco) constituye un mayor riesgo y la falta de satisfacción familiar influencia el consumo del tabaco, motivo por el cual se debe enfatizar la importancia de fortalecer las relaciones entre los adolescentes y sus padres (Cabrera Mateos et al., 2005), y mejorar la comunicación como estrategias para reducir la influencia de los compañeros que fuman en un ambiente de consumo de bebidas alcohólicas (Prinstein et al., 2001).

El consumo de tabaco es empleado por los estudiantes entrevistados como estrategia de afrontamiento, de tal forma que se logran manejar y reducir las emociones negativas que son ocasionadas por conflictos interpersonales o por el componente académico del semestre (Joseph et al., 2003) que les genera estrés, como son las actividades de seguimiento o los exámenes, dado que se busca mejorar o mantener el promedio académico; y al mismo tiempo existen otras causas como las relaciones sociales, la sobrecarga de actividades o la falta de control frente al propio entorno educativo; esto es similar a lo reportado por Panaino et al., (2014) en diferentes grupos poblacionales, quienes determinaron la existencia de circunstancias asociadas a períodos de mayor estrés y que al final pueden afectar la 
obtención de un título universitario, condiciones conflictivas con personas de su entorno o el no alcanzar el objetivo propuesto.

\section{Conclusiones}

El nivel de la problemática que se está analizando no es de máximo riesgo dado que más del $50 \%$ de los estudiantes presentan dependencia baja a la nicotina. No obstante, las instituciones de educación deben orientar sus políticas de bienestar hacia la disminución de los factores que incentiven el consumo y promover factores protectores para evitar el desarrollo de trastornos por consumo de sustancias.

Se observa en la muestra estudiada ausencia de relación entre el grado de motivación para la cesación del consumo de tabaco y el nivel de dependencia; los sujetos de la muestra presentan un nivel dudoso de dejación del consumo de tabaco a pesar de encontrarse en una dependencia muy baja; por otro lado, se puede afirmar que el factor familiar y social se convierte en promotor de la dependencia e iniciación.

A partir de los resultados se identifica una baja motivación para la dejación del consumo; por lo tanto, se debe promover intervenciones efectivas para la atención del tabaquismo contemplando desde la consejería breve hasta modelos más complejos que incluyan tratamientos combinados (intervención cognitivo-conductual, entrevista motivacional para dejar de fumar y terapia farmacológica).

El estudio presentó sesgo de participación dado que esta fue voluntaria, pues no todos los fumadores de este programa accedieron a ser incluidos.

\section{Referencias}

Alfonso, J. P., Huedo-Medina, T. B., \& Espada, J. P. (2009). Factores de riesgo predictores del patrón de consumo de drogas durante la adolescencia. Anales de Psicología, 25(2), 330-338. https://psycnet.apa.org/ record/2009-22893-015

Cabrera Mateos, J. L., Báez Álvarez, A., Gutiérrez Riquelme, F., Toledo Perdomo, I., \& Mendoza Luzardo, M. C. (2005). Prevalencia y características del consumo de tabaco en adolescentes de Lanzarote. Semergen, 31(7), 307-313. https://doi. org/10.1016/S1138-3593(05)72937-3

Castaño-Castrillón, J. J., Páez Cala, M. L., Pinzón Montes, J. H., Rojo Bustamante, E., Sánchez-Castrillón, G. A., Torres Ríos, J. M., Valencia Gómez, M.A., García Montoya, C. D., \& Gallego Giraldo, C. Andrés. (2008). Estudio descriptivo sobre tabaquismo en la comunidad estudiantil de la universidad de Manizales. 2007. Revista de La Facultad de Medicina, 56(4), 302-317. http://www.scielo. org.co/pdf/rfmun/v56n4/v56n4a03.pdf

Centers for Disease Control and Prevention (US), \& U.S. Department of Health and Human Services. (2006). The Health Consequences of Involuntary Exposure to Tobacco Smoke: A Report of the Surgeon General. In Publications and Reports of the Surgeon General. Centers for Disease Control and Prevention (US). https://www. ncbi.nlm.nih.gov/books/NBK44324/

Cifuentes, T., Rodríguez, C., Suárez, C., Blanco, M., \& Prieto, L. E. (2009). Factores que influyen en el consumo de tabaco. https://intellectum.unisabana.edu. co/bitstream/handle/10818/2600/121974. pdf? sequence $=1$ 
Coleman, T., Stevenson, K., \& Wilson, A. (2002). A New Method for Describing Smokers' Consulting Behaviours Which Indicate Their Motivation to Stop Smoking: An Exploration of Validity and ReliabilityPubMed. Fam Pract, 19(2), 154-160. https:// doi.org/10.1093/fampra/19.2.154.

Conner, M., Sandberg, T., McMillan, B., \& Higgins, A. (2006). Role of anticipated regret, intentions and intention stability in adolescent smoking initiation. British Journal of Health Psychology, 11(1), 85-101. https://doi.org/10.1348/135910705X40997

de Meneses-Gaya, I. C., Zuardi, A. W., Loureiro, S. R., \& de Crippa, J. A. S. (2009). Psychometric properties of the Fagerström Test for Nicotine Dependence. Jornal Brasileiro de Pneumologia, 35(1), 73-82. https://doi.org/10.1590/s180637132009000100011

Etter, J. F. (2005). A comparison of the content-, construct- and predictive validity of the cigarette dependence scale and the Fagerström test for nicotine dependence. Drug and Alcohol Dependence, 77(3), 259-268. https://doi.org/10.1016/j. drugalcdep.2004.08.015

Feist, J., Feist, G. J., \& Roberts, T.-A. (2014). Teorías de la personalidad (8th ed.). McGraw-Hill.

Fernández, V. H., Beligoy, M. E., Lima, Y. V., \& Barissi, P. F. (2015). Smoking and spirometric values in third year medical students: cross-sectional study. Medwave, 15(3), e6124. https://doi.org/10.5867/ medwave.2015.03.6124

Fidler, J. A., Shahab, L., \& West, R. (2011). Strength of urges to smoke as a measure of severity of cigarette dependence:
Comparison with the Fagerström Test for Nicotine Dependence and its components. Addiction, 106(3), 631-638. https://doi. org/10.1111/j.1360-0443.2010.03226.x

Fu, M., Martínez-Sánchez, J. M., López, M. J., Nebot, M., Raich, A., \& Fernández, E. (2011). Nicotine dependence and readiness to quit smoking in the Spanish population. Adicciones, 23(2), 103-109. https://doi. org/10.20882/adicciones. 153

Globocan. (2018). Cancer Today. https://gco. iarc.fr/

Gonzálvez, M. T., Espada, J. P., \& Orgilés, M. (2015). Estado de ánimo y consumo de tabaco en una muestra de adolescentes españoles. Revista Latinoamericana de Psicologia, 47(2), 86-92. https://doi.org/10.1016/j. rlp.2015.05.001

Hang, B., Sarker, A. H., Havel, C., Saha, S., Hazra, T. K., Schick, S., Iii, P. J., Rehan, V. K., Chenna, A., Divya, S., Sleiman, M., Destaillats, H., \& Gundel, L. A. (2013). Thirdhand smoke causes DNA damage in human cells. Mutagenesis, 28(4), 381. https://doi.org/10.1093/mutage/get013

Heatherton, T. F., Kozlowski, L. T., Frecker, R. C., \& Fagerstrom, K. (1991). The Fagerström Test for Nicotine Dependence: a revision of the Fagerstrom Tolerance Questionnaire. British Journal of Addiction, 86(9), 1119-1127. https://doi. org/10.1111/j.1360-0443.1991.tb01879.x

John, U., Meyer, C., Schumann, A., Hapke, U., Rumpf, H.-J., Adam, C., Alte, D., \& Lüdemann, J. (2004). A short form of the Fagerström Test for Nicotine Dependence and the Heaviness of Smoking Index in two adult population samples. Addictive Behaviors, 29(6), 1207-1212. https://doi. org/10.1016/j.addbeh.2004.03.019 
Joly, B., Perriot, J., D’Athis, P., Chazard, E., Brousse, G., \& Quantin, C. (2017). Success rates in smoking cessation: Psychological preparation plays a critical role and interacts with other factors such as psychoactive substances. PLoS ONE, 12(10). https://doi. org/10.1371/journal.pone.0184800

Joseph, S., Manafi, E., Iakovaki, A. M., \& Cooper, R. (2003). Personality, smoking motivation, and self-efficacy to quit. Personality and Individual Differences, 34(5), 749-758. https://doi.org/10.1016/ S0191-8869(02)00068-5

Kendler, K. S., Neale, M. C., Sullivan, P., Corey, L. A., Gardner, C. O., \& Prescott, C. A. (1999). A population-based twin study in women of smoking initiation and nicotine dependence. Psychological Medicine, 29(2), 299-308. https://doi.org/10.1017/ S0033291798008022

Kotz, D., Brown, J., \& West, R. (2013). Predictive validity of the Motivation To Stop Scale (MTSS): A single-item measure of motivation to stop smoking. Drug and Alcohol Dependence, 128(1-2), 15-19. https://doi. org/10.1016/j.drugalcdep.2012.07.012

Lazuras, L., Chatzipolychroni, E., Rodafinos, A., \& Eiser, J. R. (2012a). Social cognitive predictors of smoking cessation intentions among smoker employees: The roles of anticipated regret and social norms. Addictive Behaviors, 37(3), 339-341. https:// doi.org/10.1016/j.addbeh.2011.11.008

Lazuras, L., Chatzipolychroni, E., Rodafinos, A., \& Eiser, J. R. (2012b). Social cognitive predictors of smoking cessation intentions among smoker employees: The roles of anticipated regret and social norms. Addictive Behaviors, 37(3), 339-341. https:// doi.org/10.1016/j.addbeh.2011.11.008
León, F.-J., Orlandoni-Merli, G., Bernal-Luna, Y.-C., Gómez Balaguera, F. R., \& Amaya Díaz, L. P. (2019). Consumo de tabaco en estudiantes universitarios: motivación a la cesación y dependencia. Un análisis factorial (XXIX Simposio Internacional de Estadística, Ed.). Simposio Internacional de Estadística.

Organización Mundial de la Salud. (2020). Ofrecer ayuda para dejar el hábito. https:// www.who.int/tobacco/mpower/offer/es/ index 1.html

Panaino, E. F., Soares, C. B., \& Campos, C. M. S. (2014). Contextos de início do consumo de tabaco em diferentes grupos sociais. Revista Latino-Americana de Enfermagem, 22(3), 379-385. https://doi.org/10.1590/01041169.3205 .2427

Pérez-Milena, A., Martínez-Fernández, M. L., Pérez-Milena, R., Jiménez-Pulido, I., LealHelmling, F. J., \& Mesa-Gallardo, I. (2006). Tabaquismo y adolescentes: ¿Buen momento para dejar de fumar? Relación con factores sociofamiliares. Atencion Primaria, 37(8), 452-456. https://doi.org/10.1157/13088886

Pérez-Milena, A., Martínez-Fernández, M. L., Redondo-Olmedilla, M., Álvarez Nieto, C., Jiménez Pulido, I., \& Mesa Gallardo, I. (2012). Motivaciones para el consumo de tabaco entre los adolescentes de un instituto urbano. Gaceta Sanitaria, 26(1), 51-57. https://doi.org/10.1016/j. gaceta.2011.03.021

Piñeiro, B., López-Durán, A., del Río, E. F., Martínez, Ú., Brandon, T. H., \& Becoña, E. (2016). Motivation to quit as a predictor of smoking cessation and abstinence maintenance among treated Spanish smokers. Addictive Behaviors, 53, 40-45. https://doi. org/10.1016/j.addbeh.2015.09.017 
Pinilla-Vásquez, C., \& Angarita-Fonseca, A. (2012). Conocimientos y actividades asociadas al inicio del hábito de fumar durante la vida universitaria. Hacia la Promoción de la Salud. Hacia La Promoción de La Salud, 17(2), 25-39. http://www.scielo.org.co/pdf/ hpsal/v17n2/v17n2a03.pdf

Prinstein, M. J., Boergers, J., \& Spirito, A. (2001). Adolescents' and Their Friends' Health-Risk Behavior: Factors That Alter or Add to Peer Influence. Journal of Pediatric Psychology, 26(5), 287-298. https://doi. org/10.1093/JPEPSY/26.5.287

Prochaska, J. O., \& DiClemente, C. C. (1983). Stages and processes of self-change of smoking: Toward an integrative model of change. Journal of Consulting and Clinical Psychology, 51(3), 390-395. https://doi. org/10.1037/0022-006X.51.3.390

Radzius, A., Epstein, D. H., Gorelick, D. A., Cadet, J. L., Uhl, G. E., Moolchan, E. T., \& Gallo, J. J. (2003). A factor analysis of the Fagerstrom Test for Nicotine Dependence (FTND). Nicotine \& Tobacco Research, 5(2), 255-260. https://doi. org/10.1080/1462220031000073289

Raherison, C., Marjary, A., Valpromy, B., Prevot, S., Fossoux, H., \& Taytard,A. (2005). Evaluation of smoking cessation success in adults. Respiratory Medicine, 99(10), 1303-1310. https://doi.org/10.1016/j. rmed.2004.12.002

Revelle, W. (2020). Procedures for Psychological, Psychometric, and PersonalityResearch $[R$ package psych version 1.9.12.31] (1.9.12.31). Comprehensive $\mathrm{R}$ Archive Network (CRAN). https://CRAN.R-project.org/ package $=$ psych
Richmond, R. L., Kehoe, L. A., \& Webster, I. W. (1993). Multivariate models for predicting abstention following intervention to stop smoking by general practitioners. Addiction, 88(8), 1127-1135. https://doi. org/10.1111/j.1360-0443.1993.tb02132.x

Roa-Cubaque, M. A., Parada-Sierra, Z. E., Albarracín-Guevara, Y., Alba-Castro, E., Aunta-Piracon, M., \& Ortiz-León, M. (2016). Validación del test de Fagerström para adicción a la nicotina (FTND). Revista Investigación En Salud Universidad de Boyacá, 3(2), 161. https://doi. org/10.24267/23897325.185

Rodríguez Gázquez, M. de los Á., Pineda Botero, S. A., \& Vélez Yépes, L. Fernanda. (2010). Características del consumo de tabaco en estudiantes de enfermería de la Universidad de Antioquia (Colombia). Invest. Educ. Enferm, 28(3), 370-383. http://www.scielo.org.co/pdf/iee/v28n3/ v28n3a08.pdf

Romero, E., Domínguez, B., \& Castro, M. A. (2017). Predicting smoking among young people: Prospective associations from earlier developmental stages. In Revista de Psicología Clínica con Niños y Adolescentes (Vol. 4, Issue 2). Espada Sánchez, José Pedro. www.revistapcna.com

Sepúlveda-Sánchez, J. M., Canca-Sánchez, J. C., Rivas-Ruiz, F., Martín-García, M., Lorente Márquez, C., \& Timonet-Andreu, E. M. (2018). Assessing motivation to smoking cessation in hospitalized patients. Enfermeria Clinica, 28(1), 1-6. https://doi. org/10.1016/j.enfcli.2016.11.005

Services, U. S. D. ofH. and H., Prevention, C. for D. C. and, Promotion, N. C. for C. D. P. and H., \& Health, O. on S. and. (2014). Executive Summary (The Health Consequences of 
Smoking -50 Years of Progress: A Report of the Surgeon General). 1-36. https://doi. org/NBK179276

Ussher, M., Kakar, G., Hajek, P., \& West, R. (2016). Dependence and motivation to stop smoking as predictors of success of a quit attempt among smokers seeking help to quit. Addictive Behaviors, 53, 175-180. https:// doi.org/10.1016/j.addbeh.2015.10.020

Valles Vegas, C., Sanz, J., Santos García, A., \& Vicario Juan, M. (2011). ¿Son útiles los cuestionarios para valorar el hábito tabáquico en el manejo del paciente periodontal?: Estudio piloto. Periodoncia y Osteointegración, 21(1), 45-51. https://dialnet.unirioja.es/servlet/ articulo? codigo $=3892233$

Villena Ferrer, A. (2009). Factores asociados al consumo de Tabaco en Adolescentes. Rev Clin Med Fam, 2(7), 320-325. http:// scielo.isciii.es/scielo.php?pid=S1699$695 \mathrm{X} 2009000200002 \& \mathrm{script}=\mathrm{sci}$ abstract\&tlng=pt

Warren, C. W., Sinha, D. N., Lee, J., Lea, V., \& Jones, N. R. (2011). Tobacco use, exposure to secondhand smoke, and cessation counseling among medical students: cross-country data from the Global Health Professions Student Survey (GHPSS), 2005-2008. BMC Public Health, 11, 72. https://doi.org/10.1186/14712458-11-72

World Health Organization. (2004). Neuroscience of psychoactive substance use and dependence. https://www.who. int/substance_abuse/publications/en/ Neuroscience.pdf

Yang, T., Yu, L., Bottorff, J. L., Wu, D., Jiang, S., Peng, S., \& Young, K. J. (2015). Global Health Professions Student Survey (GHPSS) in Tobacco Control in China. American Journal of Health Behavior, 39(5), 732-741. https://doi.org/10.5993/AJHB.39.5.14 